\title{
Mössbauer spectroscopy of the iron cores in human liver ferritin, ferritin in normal human spleen and ferritin in spleen from patient with primary myelofibrosis: preliminary results of comparative analysis
}

\author{
M. I. Oshtrakh • I. V. Alenkina • \\ A. V. Vinogradov $\cdot$ T. S. Konstantinova \\ E. Kuzmann • V. A. Semionkin
}

Received: 23 August 2012/Accepted: 18 December 2012/Published online: 5 March 2013

(C) Springer Science+Business Media New York 2013

\begin{abstract}
Comparative study of human liver ferritin and spleen tissues from healthy human and patient with primary myelofibrosis was carried out using Mössbauer spectroscopy with a high velocity resolution at 295 and $90 \mathrm{~K}$ and with a low velocity resolution at $20 \mathrm{~K}$. The results obtained demonstrated that the iron content in patient's spleen in the form of iron storage proteins was about ten times larger than that in normal tissue. However, in the case of patient with primary myelofibrosis the magnetic anisotropy energy barrier differed from that in normal case and, probably, the iron core size was supposed to be slightly larger than that in both normal spleen tissue and normal human liver ferritin in contrast to well-known
\end{abstract}

M. I. Oshtrakh · I. V. Alenkina · V. A. Semionkin Department of Physical Techniques and Devices for Quality Control, Institute of Physics and Technology, Ural Federal University, Ekaterinburg 620002, Russian Federation

M. I. Oshtrakh ( $₫)$ · I. V. Alenkina · V. A. Semionkin Department of Experimental Physics, Institute of Physics and Technology, Ural Federal University, Ekaterinburg 620002, Russian Federation

e-mail: oshtrakh@mail.utnet.ru

\section{A. V. Vinogradov - T. S. Konstantinova}

Ural State Medical Academy, Repin Str., 3, Ekaterinburg 620028, Russian Federation

\section{E. Kuzmann}

Institute of Chemistry, Eötvös Loránd University,

Budapest, Hungary data for iron overload in patients with thalassemia accompanied by the iron-core size increase. Therefore, the iron overload in the case of patient with primary myelofibrosis may be related to increase in the ferritin content mainly. It was also found that Mössbauer hyperfine parameters for normal and patient's spleen and normal human liver ferritin demonstrated some small differences related, probably, to some small structural variations in the ferritin iron cores of patient's spleen.

Keywords Iron storage proteins - Spleen tissues . Mössbauer spectroscopy $\cdot$ Primary myelofibrosis

\section{Introduction}

Ferritin, an iron storage protein, contains nanosized iron core in the form of ferrihydrite type complex which demonstrates variations in different tissues, different animals and in normal and pathological cases. This iron core has an average composition of $5 \mathrm{Fe}_{2} \mathrm{O}_{3} \times 9 \mathrm{H}_{2} \mathrm{O}$ or can be considered as hydrous ferric oxide complex with an approximate formula of $(\mathrm{FeOOH})_{8}\left(\mathrm{FeO}: \mathrm{OPO}_{3} \mathrm{H}_{2}\right)$ (Theil 1987). A central cavity for polynuclear iron core in ferritin has a size of 5-8 $\mathrm{nm}$. The iron core may contain up to 4,500 $\mathrm{Fe}(\mathrm{III})$ atoms, however, the average number of iron atoms in ferritin found in tissues is usually varied between 1,000 and 2,500 (Chasteen and Harrison 1999). The largest ferritin content can be found in liver 
and spleen tissues. In humans total body iron stores are maintained normally within the range of 200-1,500 mg (Piperno 1998). However, there are a number of diseases, so called "iron overload disease" related to increase in total body iron stores. Iron overload can be classified as primary or secondary depending whether it results from a primary defect in the regulation of iron balance or is secondary to other genetic or acquired disorders (see Piperno 1998; Fleming and Ponka 2012; Siddique and Kowdley 2012). The presence of iron in iron storage proteins permits to apply Mössbauer spectroscopy for analysis of the iron content and state in these proteins and tissues containing ferritin (see, for instance, St. Pierre et al. 1996, 2000; Webb et al. 1992, 1996, 1999; Papaefthymiou 2010). Sensitivity of Mössbauer spectroscopy to the magnetic state of iron containing nanoparticles appeared to be useful for evaluation of the iron core size in ferritin (Dickson 1999; Papaefthymiou 2009). Mössbauer spectra of iron storage proteins both purified and in tissues, demonstrated appearance of magnetically split components (magnetic sextets) in addition to paramagnetic doublets (quadrupole doublets) with temperature decrease in the range of 300-4.2 K. It was found that increase in the area of magnetic component with decrease in the area of paramagnetic component related to the size of nanoparticles, i.e. the less nanoparticle size corresponded to the lower temperature of magnetic component appearance. This feature was used in various studies of iron overload diseases. One of the wellknown examples of the study of tissues from patients with secondary iron overload was investigation of spleen from patients with $\beta$-thalassemia (see, for instance, St. Pierre et al. 1998, 2000; Hackett et al. 2007). In these studies the authors demonstrated that Mössbauer spectra of patients' spleen tissues measured at room temperature consisted of paramagnetic doublet while those measured at $78 \mathrm{~K}$ consisted of paramagnetic doublet and magnetic sextet. In contrast, Mössbauer spectra of normal spleen measured at $78 \mathrm{~K}$ consisted of paramagnetic doublet only. This finding can be explained as increase in the iron core size in the iron storage proteins as well as complicated iron core structure with the presence of ferrihydrite and other ferric hydrous oxide forms.

Primary myelofibrosis (PMF) is a myeloproliferative neoplasm, the classical clinical phenotype being featured by leukoerythroblastic anemia, bone marrow fibrosis, enlargement of the spleen and liver, etc. (see, for instance, Hasselbalch 2009; Tefferi 2011). Clinically, PMF is characterized in part by anemia, multi-organ extramedullary hematopoiesis that often involves the spleen and liver, and so on (Mesa et al. 2006). Therefore, it is interesting to test the iron overload in spleen tissue at this pathology and compare with known secondary iron overload spleen data. In this study we applied Mössbauer spectroscopy with a high velocity resolution for measurements at 295 and $90 \mathrm{~K}$ and conventional Mössbauer spectroscopy for measurements at $20 \mathrm{~K}$. It should be noted that Mössbauer spectroscopy with a high velocity resolution demonstrated more possibilities for detailed analysis of studied biological materials (see Oshtrakh et al. 2010, 2011b, 2011c; Oshtrakh and Semionkin 2013).

\section{Materials and methods}

Sample preparation and characterization

Samples of human spleen tissues were obtained at the Hematological Division of the Sverdlovsk Regional Clinical Hospital No. 1 (Ekaterinburg, Russian Federation) within the human rights and ethical guidelines. The spleen samples were obtained from a patient with primary myelofibrosis with myeloid metaplasia after splenectomy and a healthy man after traumatic spleen lesion. The diagnosis and classification of PMF was made according to the World Health Organization criteria (see Tefferi 2011). Thin sections of both spleen samples were prepared for histochemical and histological analysis using hematoxylin and eosin stain for normal spleen and Van Gieson's stain for patient's spleen as well as Prussian Blue or Perls' reaction was used for both spleen sections for iron detection. These sections were characterized using optical microscopy. Spleen samples were washed from blood using physiological solution then lyophilized. For further investigations lyophilized spleen samples were powdered. For comparison we used also powder of lyophilized normal human liver ferritin provided by the Department of Biochemistry, Russian State Medical University, Moscow (see Oshtrakh et al. 2001). These powders were analyzed by transmission electron microscopy (TEM) using MORGAGNI 268D transmission electron microscope 
(Chemical Research Center, HAS, Budapest). Samples for Mössbauer spectroscopy were prepared using sample holders with diameter of $20 \mathrm{~mm}$ and height of $9 \mathrm{~mm}$ for spleen powders with a weight of 900-1,100 mg of powdered tissues while for ferritin sample we used $100 \mathrm{mg}$ of protein in the holder with protein layer thickness up to $2 \mathrm{~mm}$.

\section{Mössbauer spectra measurement}

Mössbauer spectra of spleen and ferritin samples were measured at the Ural Federal University, Ekaterinburg using an automated precision Mössbauer spectrometric system on the base of the spectrometer SM-2201 with a high velocity resolution and temperature variable liquid nitrogen cryostat with moving absorber. Details and characteristics of this equipment and the system were given elsewhere (Oshtrakh et al. 2009; Semionkin et al. 2010; Oshtrakh and Semionkin 2013). The $\sim 1.8 \times 10^{9} \mathrm{~Bq}{ }^{57} \mathrm{Co}$ in rhodium matrix source was used at room temperature. The spectra of both spleen and ferritin samples were measured at $295 \mathrm{~K}$ while the patient's spleen and ferritin samples were also measured at $90 \mathrm{~K}$. The Mössbauer spectra were measured in transmission geometry with moving absorber in the cryostat and recorded in 4,096 channels. The temperature stability in cryostat was $\pm 1 \mathrm{~K}$. For their analysis, the spectra of spleen samples were converted into 1,024 channels by a consequent summation of four neighboring channels while spectra of ferritin sample were analyzed as measured (in 4,096 channels). Statistical rate in the normal spleen spectrum was $6.4 \times 10^{6}$ counts per channel with a signalto-noise ratio of 8 while that for patient's spleen spectra were $5.7 \times 10^{5}$ counts per channel with a signal-to-noise ratio of 22 at $295 \mathrm{~K}$ and $3.7 \times 10^{6}$ counts per channel with a signal-to-noise ratio of 58 at $90 \mathrm{~K}$ (spectra were measured up to 4 weeks). Statistical rates in the ferritin spectra were $1.05 \times 10^{6}$ counts per channel with a signal-to-noise ratio of 69 at $295 \mathrm{~K}$ and $\sim 3.2 \times 10^{5}$ counts per channel with a signal-to-noise ratio of 44 at $90 \mathrm{~K}$ (spectra were measured up to 1 week).

Additionally, Mössbauer spectra of both spleen and ferritin samples were measured at the Eötvös Loránd University, Budapest with a low velocity resolution using the KFKI type conventional spectrometer with APD closed cycle refrigerator at $20 \mathrm{~K}$. The $\sim 2.5 \times 10^{9}$ $\mathrm{Bq}{ }^{57} \mathrm{Co}$ in rhodium matrix source was used at room temperature. The statistical counting rates for the 250 channels spectra of normal human and patient's spleen were $6.0 \times 10^{6}$ counts per channel with the signal-tonoise ratio of 15 and $5.7 \times 10^{6}$ counts per channel with the signal-to-noise ratio of 32 , respectively, while that for the human liver ferritin spectrum was $\sim 3.7 \times 10^{6}$ counts per channel with the signal-to-noise ratio of 74 (spectra were measured up to 3 days).

The main differences between Mössbauer spectrometers are in velocity mode (a saw-tooth shape velocity reference signal formed by 4,096 bits in SM2201 and a triangular velocity reference signal formed by 1,024 bits in KFKI) as well as in different quality of the velocity driving system. Therefore, spectra registered using KFKI spectrometer on the direct and reverse motion and further folded have significantly larger error on velocity scale than those measured using SM-2201. Nevertheless, this spectrometer is very useful for $20 \mathrm{~K}$ measurements of studied samples.

The standard absorbers of $\alpha$-Fe foil with a thickness of $10 \mu \mathrm{m}$ and sodium nitroprusside with a thickness of
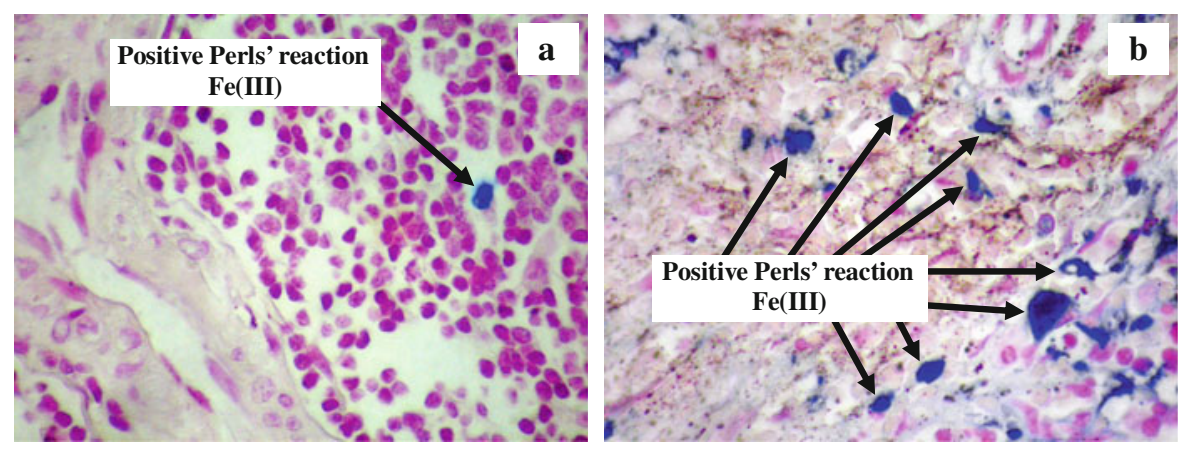

Fig. 1 Optical micrographs $(\times 400)$ of parenchyma cells in normal human spleen $(\mathbf{a})$ and spleen from patient with primary myelofibrosis (b). Cells containing Fe(III) are colored in blue after Perls' reaction 
$5 \mathrm{mg} \mathrm{Fe} / \mathrm{cm}^{2}$ were used for velocity calibration of both spectrometers for a large and a small velocity ranges, respectively. All measured spectra were computer fitted with the least squares procedure using UNIVEM-MS program with Lorentzian line shape. Spectral parameters such as: isomer shift, $\delta$, quadrupole splitting, $\Delta \mathrm{E}_{\mathrm{Q}}$, magnetic hyperfine field, $\mathrm{H}_{\mathrm{eff}}$, linewidth, $\Gamma$, relative subspectrum area, $S$, and statistical quality of the fit, $\chi^{2}$, were determined. In the case of the high velocity resolution spectrometer SM-2201 an instrumental error for the velocity scale or systematic error for the each spectra point was \pm 0.5 channel, instrumental error for hyperfine parameters evaluation was \pm 1 channel while instrumental error for $\Gamma$ evaluation was \pm 2 channels. It should be noted that spectrometer characteristics determined an integral velocity error (total mechanical and electronics systematic and random errors) which was several times less than a half of channel value in $\mathrm{mm} / \mathrm{s}$ during spectra measurements using 4,096 channels (Oshtrakh et al. 2009). If error calculated with the fitting procedure (fitting error) for these parameters exceeded the instrumental (systematic) error, we used the larger error. Velocity resolution (velocity per one channel) in the high velocity resolution Mössbauer spectra was $\sim 0.005 \mathrm{~mm} / \mathrm{s}$ per channel for the $295 \mathrm{~K}$ spectra of spleen samples, $0.022 \mathrm{~mm} / \mathrm{s}$ per channel for the $90 \mathrm{~K}$ spectrum of patient's spleen and $0.001 \mathrm{~mm} / \mathrm{s}$ per channel for both 295 and $90 \mathrm{~K}$ ferritin spectra. For the low velocity resolution Mössbauer spectra an instrumental (systematic) error for a velocity scale after folding was at least \pm 1 channel for each spectrum point, the systematic error for the hyperfine parameters was \pm 2 channel while that for a line width was \pm 4 channels. A velocity resolution in the spectra after folding was $\sim 0.11 \mathrm{~mm} / \mathrm{s}$ per channel. The relative error for S did not exceed $10 \%$. Criteria of the best fit were differential spectra, $\chi^{2}$ values and physical meaning of parameters. The value of standard deviation $(\sigma)$ of $\chi^{2}$ was 0.044 for spectra presented in 1,024 channels and 0.022 for 4,096 channels. Values of $\delta$ are given relative to $\alpha$-Fe at $295 \mathrm{~K}$.

\section{Results and discussion}

Optical micrographs of parenchymal cells in normal human spleen and PMF patient's spleen are shown in

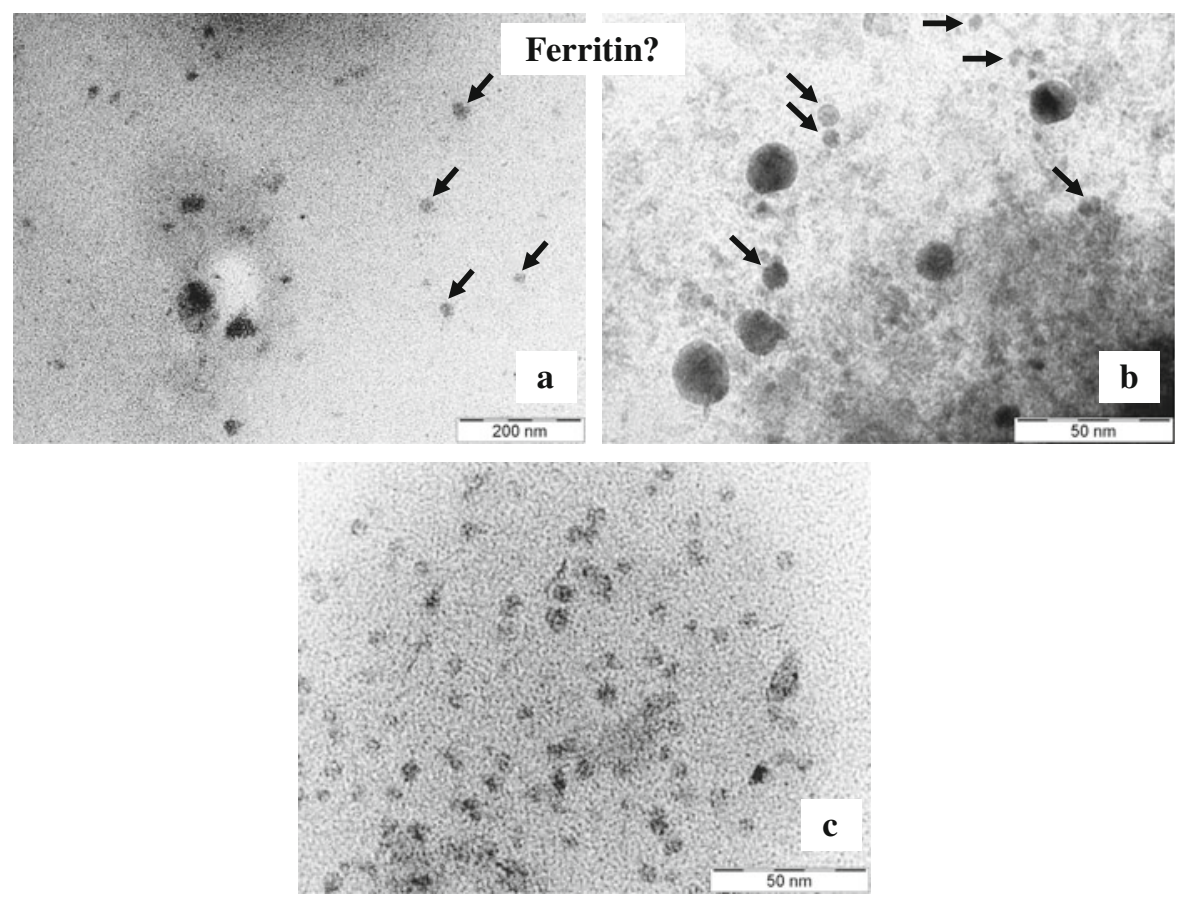

Fig. 2 Images of transmission electron microscopy of powdered normal human spleen (a), spleen from patient with primary myelofibrosis (b) and human liver ferritin (c). Possible ferritin iron cores are indicated by arrows 
Fig. 1. It is clearly seen a large amount of blue colored cells in patient's spleen. This is a result of Perls' reaction indicating the presence of $\mathrm{Fe}(\mathrm{III})$ in the cells. TEM images of spleen samples and human liver ferritin are shown in Fig. 2. Unfortunately, it was not possible to identify the iron cores in spleen samples exactly while in the sample of human liver ferritin the iron cores are clearly seen (the iron core sizes should not exceed $8 \mathrm{~nm}$ ). If we suggest that arrows indicate the iron cores in normal and patient's spleen samples we can suppose larger amount of iron storage proteins in patient's spleen with some variation in the iron core size.

Mössbauer spectra of normal human liver ferritin, normal human spleen and spleen from patient with PMF measured at room temperature with a high velocity resolution are shown in Fig. 3. These spectra represent two main peaks (paramagnetic doublet shape) with different absorption effect. In the case of human liver ferritin a large amount of iron in the sample was provided by protein extraction from the tissue. In this case concentrated ferritin in the sample demonstrated a large amount of proteins while iron content in the core may be considered as correspondent to the normal human liver iron storage. In spite of Mössbauer hyperfine parameters for liver and spleen ferritin are slightly different (Chua-anusorn et al. 1994; Oshtrakh et al. 2006, 2008) we can further use the data for human liver ferritin for comparison with spleen data. In the spectra of human normal and patient's spleen a small doublet component was found in addition to the main component. This minor component is related to residual hemoglobin (probably in oxidized form) in tissues which was also observed in previous Mössbauer studies (Chua-anusorn et al. 1994; St. Pierre et al. 1998). As for the absorption effect in both spleen spectra, it is clearly seen that the effect for patient's spleen is about ten times larger than that for normal spleen. It is well known that the absorption effect is proportional to the Mössbauer effect probability and the number of ${ }^{57} \mathrm{Fe}$ nuclei in the sample. If we suppose that the Mössbauer effect probability can be considered the same for the ferritin iron cores in both spleen samples, the number of ${ }^{57} \mathrm{Fe}$ nuclei, which natural abundance is $\sim 2.19 \%$, and total iron content in spleen from patient with PMF is about ten times larger than that in normal spleen. This finding correlates with histochemical analysis of spleen tissues and demonstrates iron overload in patient's spleen. Therefore, for further analysis we measured patient's spleen samples and human liver ferritin at $90 \mathrm{~K}$ and compared with other results (see Fig. 4). It is clearly seen that in Mössbauer spectra of spleen tissues from patients with $\beta$-thalassemia a six lines component (magnetic sextet) with different intensity can be observed in addition to paramagnetic component (St. Pierre et al. 1998). Similarly, in the Mössbauer spectrum of Imferon frozen solution (an iron-dextran complex which modeling ferritin and contain $\beta-\mathrm{FeOOH}$ iron core) measured at $90 \mathrm{~K}$ six lines components were observed in addition to paramagnetic components (Oshtrakh et al. 2011a). In contrast, Mössbauer spectra of human liver ferritin and patient's spleen demonstrated paramagnetic components only. This means that the iron core size in thalassemia spleen and Imferon was larger than that in normal human liver ferritin and spleen from patient with PMF. Then Mössbauer spectra of human liver ferritin and normal human and patient's spleen were measured at $20 \mathrm{~K}$ with a low velocity resolution (Fig. 5). It is clearly seen appearance of magnetically split component in addition to paramagnetic doublet in the spectrum of patient's spleen while in the case of normal human liver ferritin magnetic component is very weak. In the case of normal human spleen we cannot detect a magnetic component due to a very small signal-to-noise ratio and residual parabolic distortion which remains after spectra folding (see Fig. 5d-f). Therefore, the $20 \mathrm{~K}$ Mössbauer spectra were fitted taking into account residual parabolic distortion and obtained parameters we should consider as rough evaluation. In these fits the $\Gamma$ values for sextets were too large $(1.3-1.5 \mathrm{~mm} / \mathrm{s})$. Relative area of magnetic component was evaluated of $\sim 14 \%$ for normal human liver ferritin and $\sim 39 \%$ for the spleen from patient with PMF. It was also possible to evaluate magnetic hyperfine fields for human liver ferritin and patient's spleen: $378 \pm 8$ and $427 \pm 7 \mathrm{kOe}$, respectively. In the case of thalassemic spleen the authors observed magnetic component at $78 \mathrm{~K}$ with $\mathrm{H}_{\text {eff }}$ of about 470 kOe (St. Pierre et al. 1998) while the main magnetic components in the Imferon spectrum (5 and 6 in Fig. 4c) measured at $90 \mathrm{~K}$ had $\mathrm{H}_{\text {eff }}$ of about $472.0 \pm 0.6$ and $485.2 \pm 0.3 \mathrm{kOe}$, respectively. These findings indicate different magnetic anisotropy energy barriers for the nanosized iron cores. If we suggest similar magnetic anisotropy constant in these samples, the iron core sizes in thalassemic spleen and 

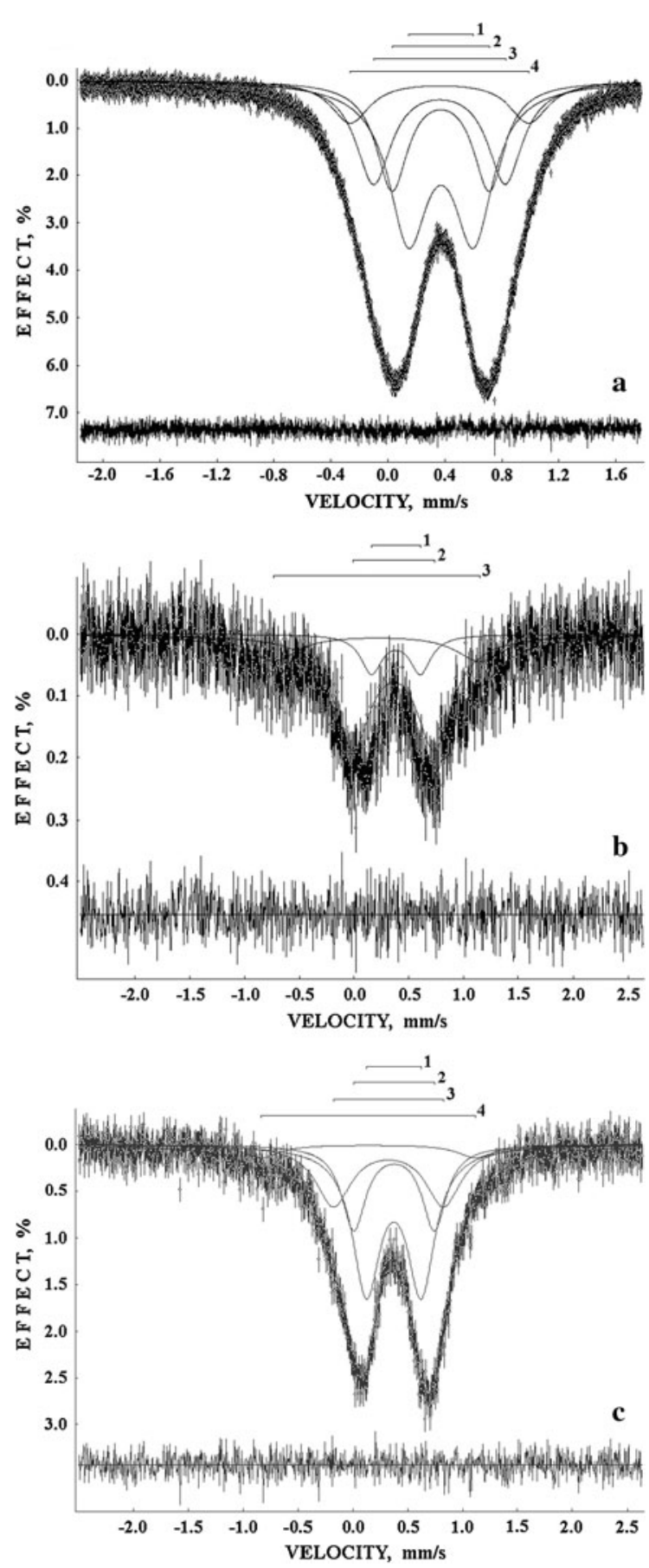

Fig. 3 Mössbauer spectra of human liver ferritin measured in 4,096 channels (a), normal human spleen presented in 1,024 channels (b) and spleen from patient with primary myelofibrosis presented in 1,024 channels (c). Indicated components are the results of the better fits, component 3 in (b) and component 4 in (c) are related to residual hemoglobin while other components are related to the iron core structural peculiarities. The differential spectra are shown below to indicate the quality of the fit. $\mathrm{T}=295 \mathrm{~K}$
Imferon should be larger than that in human liver ferritin and spleen from normal human and patient with PMF. It should be noted that in the case of thalassemic spleen the authors suggested an increase in hemosiderin (another iron storage protein) content. The iron in hemosiderin may consist of goethite-like form which demonstrated magnetic sextet at $78 \mathrm{~K}$ while iron in ferritin was in paramagnetic state (see, for instance, Mann et al. 1988; St. Pierre et al. 1998; Webb et al. 1996). In this case we can suppose that the iron in normal human spleen and spleen from patient with PMF was stored in ferritin mainly. However, the size of the iron cores in human liver ferritin and ferritin in normal human spleen was smaller than that in ferritin in spleen from patient with PMF.

Mössbauer spectra of human liver ferritin and normal and patient's spleen measured at 295 and $90 \mathrm{~K}$ were fitted using two models: (i) homogeneous iron core (rough model) with spectra fitting using one quadrupole doublet for ferritin component, and (ii) heterogeneous iron core model with spectra fitting using several quadrupole doublets for ferritin component to reach the best fit (see Oshtrakh et al. 2011a; Alenkina et al. 2013). It was interesting to observe small variations of the hyperfine parameter (quadrupole splitting) for these samples obtained using homogeneous iron core model (rough model) in the plot of $\delta$ and $\Delta \mathrm{E}_{\mathrm{Q}}$ parameters shown in Fig. 6. The values of $\delta$ and $\Delta \mathrm{E}_{\mathrm{Q}}$ for normal human liver ferritin and normal human spleen appeared to be the same within the error (larger errors for normal human spleen parameters are related to the very small signal-to-noise ratio and lower velocity resolution in the spectrum presented in 1,024 channels). However, the difference of $\Delta \mathrm{E}_{\mathrm{Q}}$ values for both normal human live ferritin and normal human spleen from that for patient's spleen observed at $295 \mathrm{~K}$ has the same tendency at $90 \mathrm{~K}$. This finding may be a result of small structural differences in the iron cores of normal human liver and spleen ferritins and patient's spleen ferritin. Application of the heterogeneous model for the spectra fits led to significant improvement of the spectra fit in the case of good signal-to-noise ratio. The obtained parameters are shown in Table 1. Mössbauer spectra of human liver ferritin measured at 295 and $90 \mathrm{~K}$ were better fitted using four quadrupole doublets in agreement with our previous data obtained for 2,048 channels spectra Oshtrakh et al. (2011a). The room temperature spectrum of normal human spleen was fitted using two 


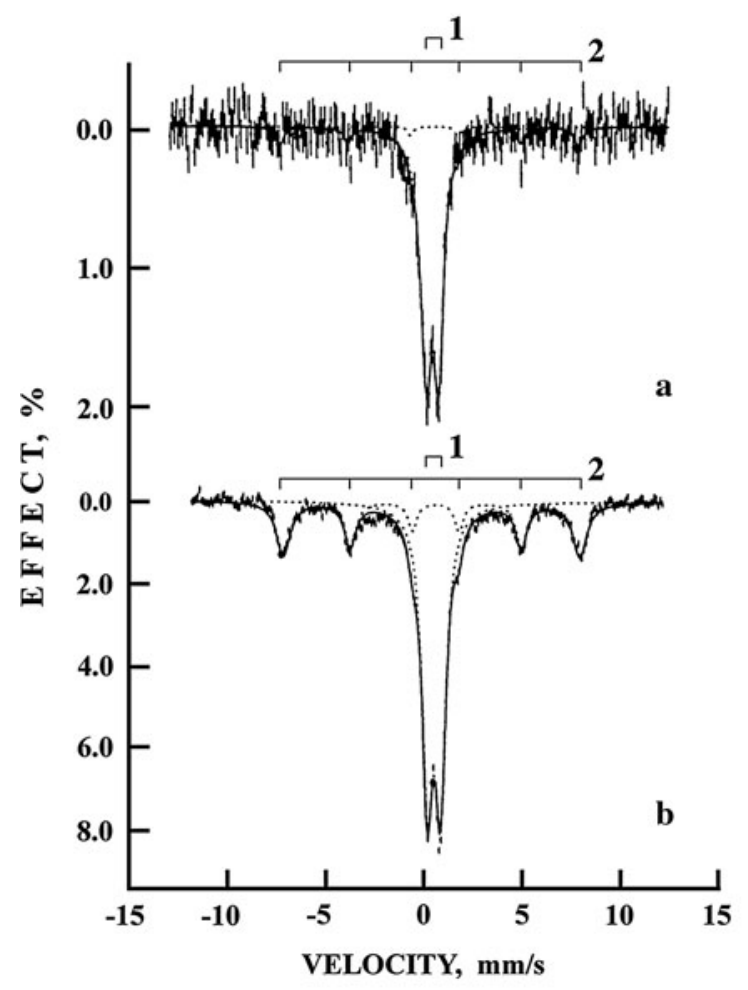

Fig. 4 Mössbauer spectra of thai $\beta$-thalassemia/hemoglobin $E$ spleen (a) and australian $\beta$-thalassemia spleen (b) measured at $78 \mathrm{~K}$ with a low velocity resolution (St. Pierre et al. 1998), Imferon frozen solution measured at $90 \mathrm{~K}$ and presented in 2,048 channels (c), and spleen from patient with primary

quadrupole doublets related to ferric oxyhydroxide for ferritin component and one doublet for the heme iron. Unfortunately, it takes significantly better signal-tonoise ratio in the spectrum to extract better and more reliable parameters. In the case of Mössbauer spectra of patient's spleen fits we obtained two sets of results related to different velocity resolution in the spectra measured at room temperature in a small velocity range and at $90 \mathrm{~K}$ in a large velocity range. The room temperature spectrum of patient's spleen was better fitted using three quadrupole doublets related to ferric
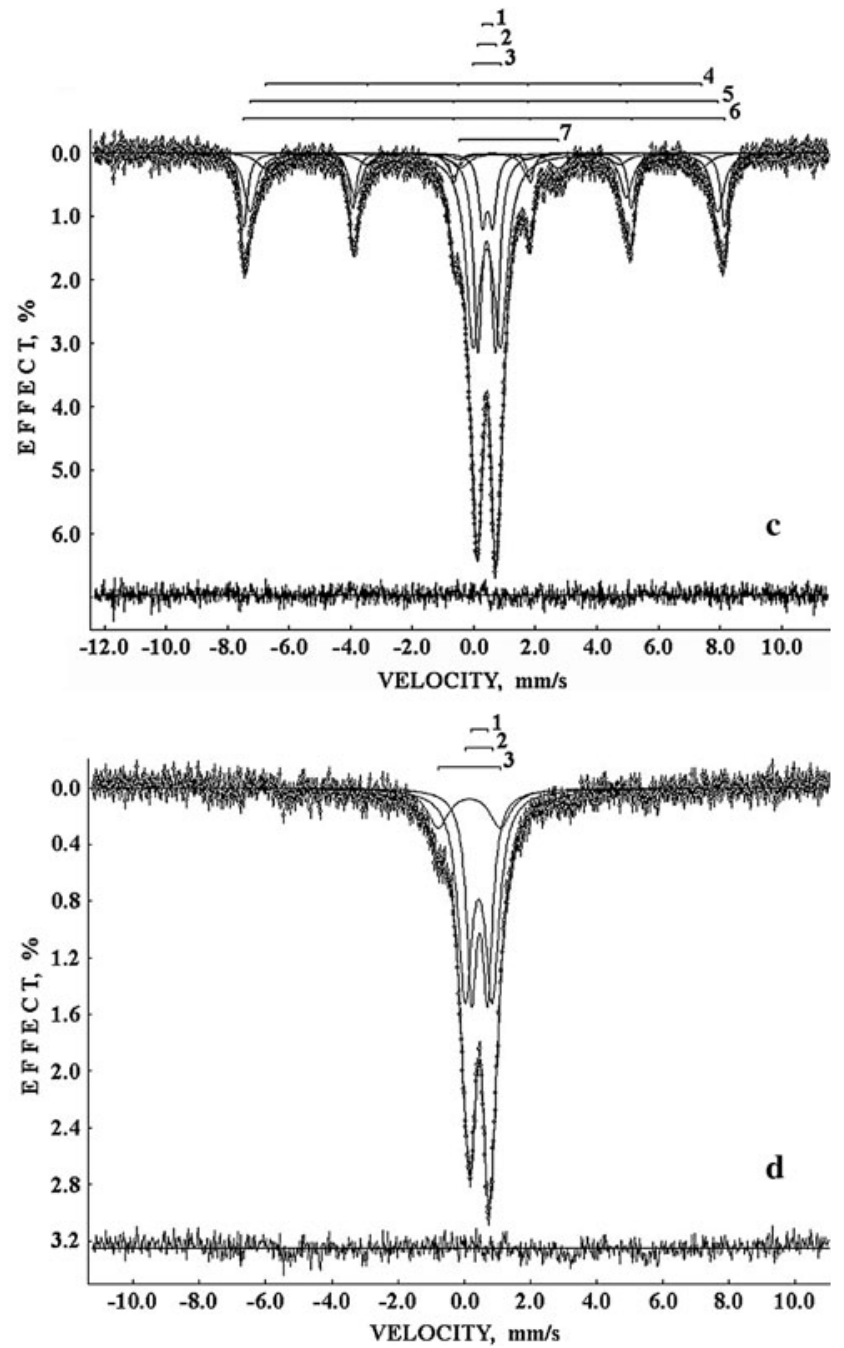

myelofibrosis measured at $90 \mathrm{~K}$ and presented in 1,024 channels (d). Indicated components are the results of the better fits. The differential spectra are shown below to indicate the quality of the fit

oxyhydroxide for ferritin component and one doublet for the heme iron while the $90 \mathrm{~K}$ one was better fitted using two quadrupole doublets related to ferric oxyhydroxide for ferritin component and one doublet for the heme iron. This is a result of differences in both signal-to-noise ratio and velocity resolution in the spectra. Nevertheless, these results confirm heterogeneous iron core model for the iron cores in iron storage proteins which in fact should be more complicated than for the core-shell model (Papaefthymiou 2009, 2010). 

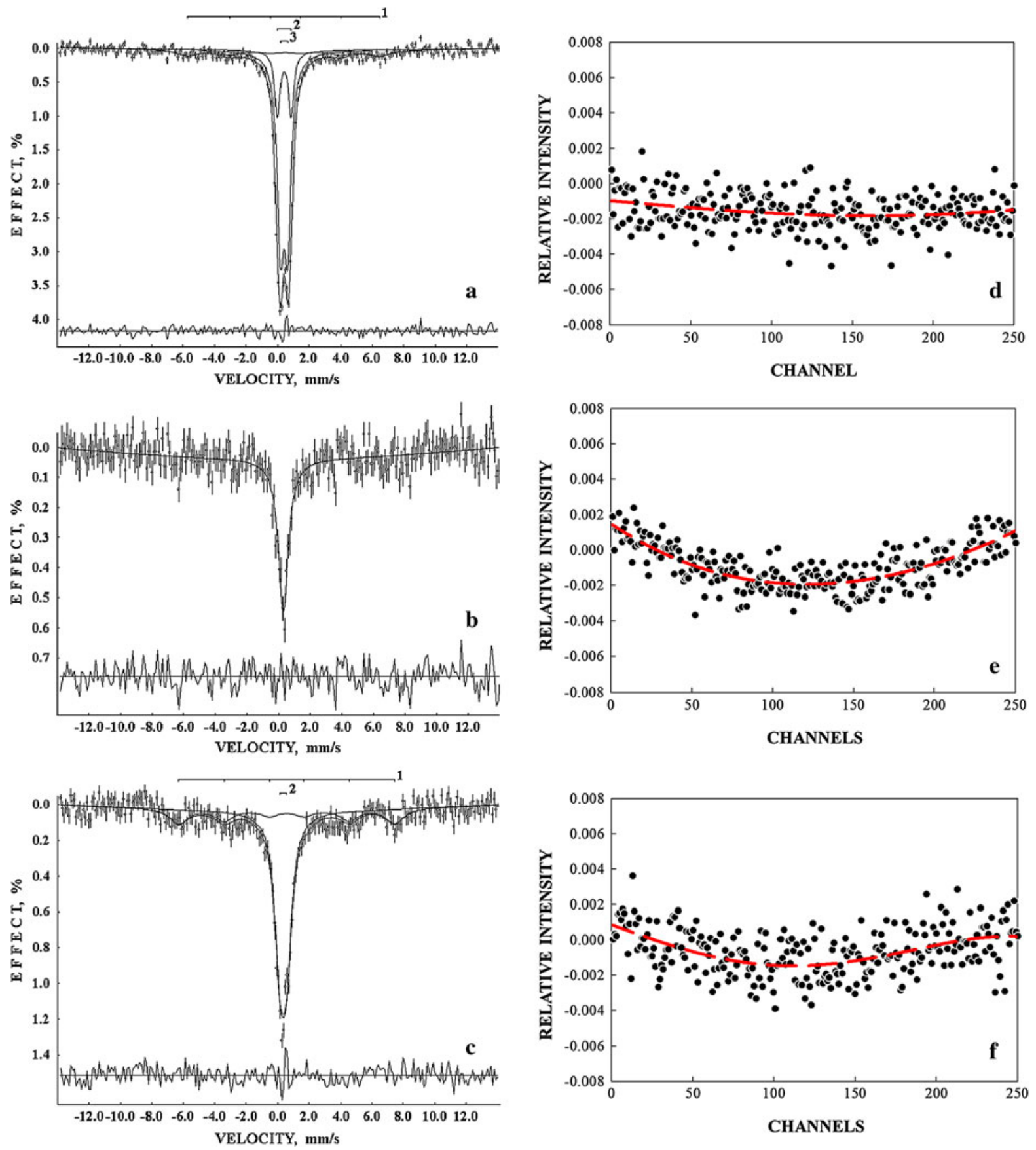

Fig. 5 Mössbauer spectra of human liver ferritin (a), normal human spleen (b) and spleen from patient with primary myelofibrosis (c) measured in 250 channels at $20 \mathrm{~K}$ and corresponded differences between the direct and reverse motion

\section{Conclusion}

The Mössbauer study of the spleen tissue from patient with primary myelofibrosis in comparison with normal

spectra for ferritin and each spleen sample (d-f, respectively). Indicated components are the results of the better fits. The differential spectra are shown below to indicate the quality of the fit

human liver ferritin and normal human spleen demonstrated significant increase in iron deposit in patient's spleen. This may be related to increased red blood cells lysis with heme iron release and 
Fig. 6 Small differences of Mössbauer hyperfine parameters for normal human liver ferritin (black circle), normal human spleen (black square) and spleen from patient with primary myelofibrosis (black triangle) obtained using the model of homogeneous iron core from Mössbauer spectra measured at $295 \mathrm{~K}$ (a) and $90 \mathrm{~K}$ (b). The error bars for normal human liver ferritin at $90 \mathrm{~K}$ are less than the symbol size

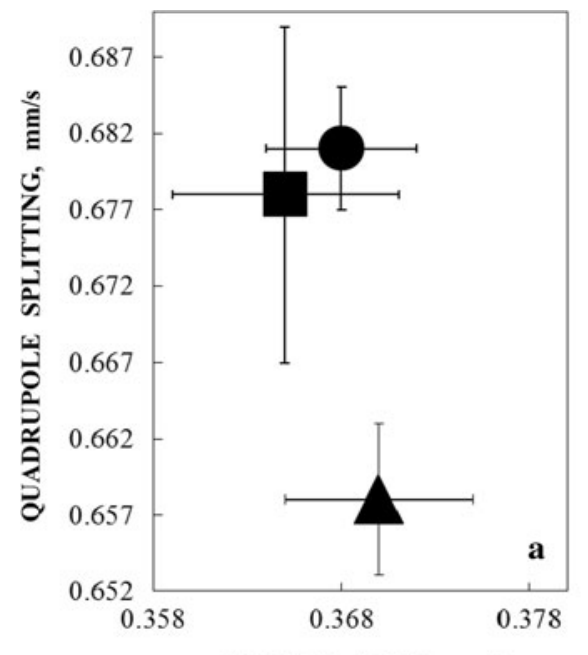

ISOMER SHIFT, $\mathrm{mm} / \mathrm{s}$

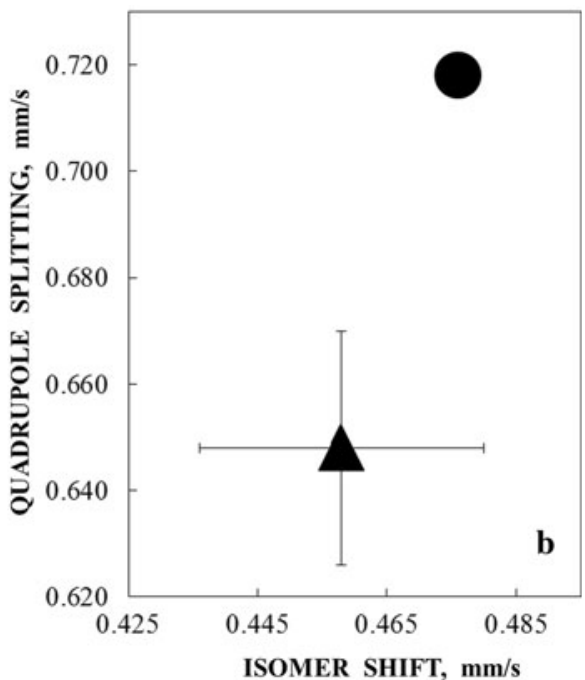

Table 1 Mössbauer parameters of normal human liver ferritin, normal human spleen and spleen from patient with primary myelofibrosis obtained from Mössbauer spectra measured with a high velocity resolution and fitted using heterogeneous iron core model

\begin{tabular}{|c|c|c|c|c|c|c|c|}
\hline Sample & $\mathrm{T}(\mathrm{K})$ & $\Gamma(\mathrm{mm} / \mathrm{s})$ & $\delta(\mathrm{mm} / \mathrm{s})$ & $\Delta \mathrm{E}_{\mathrm{Q}}(\mathrm{mm} / \mathrm{s})$ & $\mathrm{S}(\%)$ & $\chi^{2}$ & Component $^{\mathrm{a}}$ \\
\hline Normal human & 295 & $0.334 \pm 0.006$ & $0.362 \pm 0.002$ & $1.254 \pm 0.006$ & 11.2 & 1.424 & Fe-oxyhydroxide (4) \\
\hline liver ferritin & & $0.299 \pm 0.004$ & $0.361 \pm 0.001$ & $0.923 \pm 0.002$ & 24.7 & & Fe-oxyhydroxide (3) \\
\hline \multirow[t]{2}{*}{ (4,096 channels) } & & $0.270 \pm 0.002$ & $0.368 \pm 0.001$ & $0.679 \pm 0.002$ & 23.6 & & Fe-oxyhydroxide (2) \\
\hline & & $0.335 \pm 0.002$ & $0.370 \pm 0.001$ & $0.456 \pm 0.002$ & 40.5 & & Fe-oxyhydroxide (1) \\
\hline Normal human & 90 & $0.441 \pm 0.016$ & $0.458 \pm 0.003$ & $1.306 \pm 0.012$ & 11.1 & 0.985 & Fe-oxyhydroxide \\
\hline liver ferritin & & $0.389 \pm 0.007$ & $0.468 \pm 0.001$ & $0.946 \pm 0.003$ & 30.1 & & Fe-oxyhydroxide \\
\hline \multirow[t]{2}{*}{ (4,096 channels) } & & $0.356 \pm 0.005$ & $0.478 \pm 0.001$ & $0.641 \pm 0.003$ & 35.7 & & Fe-oxyhydroxide \\
\hline & & $0.365 \pm 0.008$ & $0.486 \pm 0.002$ & $0.396 \pm 0.005$ & 23.1 & & Fe-oxyhydroxide \\
\hline Normal human & 295 & $0.398 \pm 0.033$ & $0.363 \pm 0.007$ & $0.747 \pm 0.022$ & 65.2 & 0.692 & Fe-oxyhydroxide (2) \\
\hline spleen & & $0.233 \pm 0.010$ & $0.384 \pm 0.014$ & $0.447 \pm 0.034$ & 13.6 & & Fe-oxyhydroxide (1) \\
\hline (1,024 channels) & & $0.543 \pm 0.122$ & $0.208 \pm 0.029$ & $1.890 \pm 0.068$ & 21.2 & & Heme iron (3) \\
\hline Spleen from patient with PMF & 295 & $0.384 \pm 0.046$ & $0.322 \pm 0.011$ & $1.006 \pm 0.046$ & 24.4 & 0.772 & Fe-oxyhydroxide (3) \\
\hline \multirow[t]{3}{*}{ (1,024 channels) } & & $0.267 \pm 0.039$ & $0.374 \pm 0.007$ & $0.736 \pm 0.023$ & 23.8 & & Fe-oxyhydroxide (2) \\
\hline & & $0.308 \pm 0.013$ & $0.371 \pm 0.005$ & $0.501 \pm 0.016$ & 46.2 & & Fe-oxyhydroxide (1) \\
\hline & & $0.411 \pm 0.102$ & $0.142 \pm 0.029$ & $1.953 \pm 0.057$ & 5.6 & & Heme iron (4) \\
\hline Spleen from patient with PMF & 90 & $0.512 \pm 0.044$ & $0.442 \pm 0.022$ & $0.820 \pm 0.022$ & 49.9 & 1.017 & Fe-oxyhydroxide (2) \\
\hline \multirow[t]{2}{*}{ (1,024 channels) } & & $0.372 \pm 0.044$ & $0.469 \pm 0.022$ & $0.482 \pm 0.022$ & 35.5 & & Fe-oxyhydroxide (1) \\
\hline & & $0.776 \pm 0.044$ & 0.156 (fixed) & $1.877 \pm 0.036$ & 14.6 & & Heme iron (3) \\
\hline
\end{tabular}

${ }^{a}$ Numbers in parenthesis correspond to the spectral components in Figs. 3 and $4 \mathrm{~d}$

extramedullary hematopoiesis in patient's spleen. The first process leads to a larger iron storage in spleen cells to protect cells damage by ferric ions and, therefore, to increase in apoferritin synthesis in spleen. The extramedullary hematopoiesis in spleen may be related to the use of stored iron and, therefore, in larger iron storage in spleen than in normal case. Moreover, this stored iron should be easier released for hematopoiesis in spleen and, therefore, it should be stored in ferritin rather than hemosiderin. Therefore, in contrast to other iron overload like thalassemia in the case of primary myelofibrosis the patient's spleen contain 
significantly large amount of ferritin with less size of the iron core than that for thalassemic spleen. However the size of the iron core in ferritin in the spleen from patient with primary myelofibrosis is slightly larger than that in normal human liver ferritin and ferritin in normal human spleen. It was also interesting to observe small differences of quadrupole splitting for the spleen from patient with primary myelofibrosis and both normal human liver ferritin and normal human spleen obtained within the rough model of homogeneous iron core in ferritin. This finding also may be considered as a result of some differences in the iron core formation in normal ferritin and in ferritin in the spleen from patient with primary myelofibrosis. However, further studies are required for better understanding of mechanisms of the ferritin iron core formation in spleen in the case of primary myelofibrosis.

Acknowledgments The authors wish to thank Prof. P.G. Prokopenko (Department of Biochemistry, Russian State Medical University, Moscow) for the gift of human liver ferritin and Dr. S.E. Ioshchenko for histochemical analysis and optical micrographs (Sverdlovsk Regional Bureau of Morbid Anatomy, Ekaterinburg). Many thanks to Dr. Péter Németh (Chemical Research Center, HAS, Budapest) for his electron microscopy images. This work was supported by the basic financing from the Ministry of Science and Education of Russian Federation. I.V.A. is supported in part by the Ural Federal University development program for support of young scientists.

\section{References}

Alenkina IV, Oshtrakh MI, Klepova YuV, Dubiel SM, Sadovnikov NV, Semionkin VA (2013) Comparative study of the iron cores in human liver ferritin, its pharmaceutical models and ferritin in chicken liver and spleen tissues using Mössbauer spectroscopy with a high velocity resolution. Spectrochim Acta A 100:88-93

Chasteen ND, Harrison PM (1999) Mineralization in ferritin: an efficient means of iron storage. J Struc Biol 126:182-194

Chua-anusorn W, St. Pierre TG, Webb J, Macey DJ, Yansukon P, Pootrakul P (1994) Mössbauer spectroscopic study of the forms of iron in normal human liver and spleen tissue. Hyperfine Interact 91:905-910

Dickson DPE (1999) Nanostructural magnetism in living systems. J Magn Magn Mater 203:46-49

Fleming RE, Ponka P (2012) Iron overload in human disease. N Engl J Med 366:348-359

Hackett S, Chua-anusorn W, Pootrakul P, St. Pierre TG (2007) The magnetic susceptibilities of iron deposits in thalassaemic spleen tissue. Biochim Biophys Acta 1772:330-337
Hasselbalch HC (2009) Myelofibrosis with myeloid metaplasia: the advanced phase of an untreated disseminated hematological cancer-Time to change our therapeutic attitude with early upfront treatment? Leuk Res 33:11-18

Mann S, Wade VJ, Dickson DPE, Reid NMK, Ward RJ, O'Connell M, Peters TJ (1988) Structural specificity of haemosiderin iron cores in iron-overload diseases. FEBS Lett 234:69-72

Mesa RA, Barosi G, Cervantes F, Reilly JT, Tefferi A (2006) Myelofibrosis with myeloid metaplasia: disease overview and non-transplant treatment options. Best Pract Res Clin Haematol 19:495-517

Oshtrakh MI, Semionkin VA (2013) Mössbauer spectroscopy with a high velocity resolution: advances in biomedical, pharmaceutical, cosmochemical and nanotechnological research. Spectrochim Acta A 100:78-87

Oshtrakh MI, Semionkin VA, Prokopenko PG, Milder OB, Livshits AB, Kozlov AA (2001) Hyperfine interactions in the iron cores from various pharmaceutically important iron-dextran complexes and human ferritin: a comparative study by Mössbauer spectroscopy. Int J Biol Macromol 29:303-314

Oshtrakh MI, Milder OB, Semionkin VA, Malakheeva LI, Prokopenko PG (2006) An analysis of iron storage proteins in chicken liver and spleen tissues in comparison with human liver ferritin by Mössbauer spectroscopy. J Radioanal Nucl Chem 269:671-677

Oshtrakh MI, Milder OB, Semionkin VA (2008) A study of human liver ferritin and chicken liver and spleen using Mössbauer spectroscopy with high velocity resolution. Hyperfine Interact 181:45-52

Oshtrakh MI, Semionkin VA, Milder OB, Novikov EG (2009) Mössbauer spectroscopy with high velocity resolution: an increase of analytical possibilities in biomedical research. J Radioanal Nucl Chem 281:63-67

Oshtrakh MI, Semionkin VA, Milder OB, Alenkina IV, Novikov EG (2010) Biomedical application of Mössbauer spectroscopy with a high velocity resolution: revealing of small variations. Spectroscopy 24:593-599

Oshtrakh MI, Alenkina IV, Dubiel SM, Semionkin VA (2011a) Structural variations of the iron cores in human liver ferritin and its pharmaceutically important models: a comparative study using Mössbauer spectroscopy with a high velocity resolution. J Mol Struct 993:287-291

Oshtrakh MI, Alenkina IV, Milder OB, Semionkin VA (2011b) Mössbauer spectroscopy with a high velocity resolution in the study of iron-containing proteins and model compounds. Spectrochim Acta A 79:777-783

Oshtrakh MI, Berkovsky AL, Kumar A, Kundu S, Vinogradov AV, Konstantinova TS, Semionkin VA (2011c) Heme iron state in various oxyhemoglobins probed using Mössbauer spectroscopy with a high velocity resolution. Biometals 24:501-512

Papaefthymiou GC (2009) Nanoparticle magnetism. Nano Today 4:438-447

Papaefthymiou GC (2010) The Mössbauer and magnetic properties of ferritin cores. Biochim Biophys Acta 1800:886-897

Piperno A (1998) Classification and diagnosis of iron overload. Haematology 83:447-455 
Semionkin VA, Oshtrakh MI, Milder OB, Novikov EG (2010) A high velocity resolution Mössbauer spectrometric system for biomedical research. Bull Russ Acad Sci Phys 74:416-420

Siddique A, Kowdley KV (2012) The iron overload syndromes. Aliment Pharmacol Ther 35:876-893

St. Pierre TG, Chan P, Bauchspiess KR, Webb J, Betteridge S, Walton S, Dickson DP (1996) Synthesis, structure and magnetic properties of ferritin cores with varying composition and degrees of structural order: models for iron oxide deposits in iron-overload diseases. Coord Chem Rev 151:125-143

St. Pierre TG, Chua-anusorn W, Webb J, Macey DJ, Pootrakul P (1998) The form of iron oxide deposits in thalassemic tissues varies between different groups of patients: a comparison between thai $\beta$-thalassemia/hemoglobin E patients and australian $\beta$-thalassemia patients. Biochim Biophys Acta 1407:51-60

St. Pierre TG, Chua-anusorn W, Webb J, Macey DJ (2000) Iron overload diseases: the chemical speciation of non-heme iron deposits in iron loaded mammalian tissues. Hyperfine Interact 126:75-81
Tefferi A (2011) Primary myelofibrosis: 2012 update on diagnosis, risk stratification, and management. Am J Hematol 86:1018-1026

Theil EC (1987) Ferritin: structure, gene regulation, and cellular function in animals, plants, and microorganisms. Ann Rev Biochem 56:289-315

Webb J, St. Pierre TG, Macey DJ, Tran KC, Pootrakul P (1992) Biogenic ferrihydrite: effect of $\beta$-thalassaemia/haemoglobin $\mathrm{E}$ disease on the structure of ferrihydrite present in ferritins isolated from iron-loaded human heart and spleen tissue. In: Skinner HCW, Fitzpatrick RW (eds) Biomineralization processes, iron, manganese. Catena Verlag, Cremlingen, pp 169-178

Webb J, St. Pierre TG, Tran KC, Chua-anusorn W, Macey DJ, Pootrakul P (1996) Biologically significant iron(III) oxyhydroxy polymers: Mössbauer spectroscopic study of ferritin and hemosiderin in pancreas tissue of thalassemia/ hemoglobin E disease. Inorg Chim Acta 242-243:121-125

Webb J, Macey DJ, Chua-anusorn W, St. Pierre TG, Brooker LR, Rahman I, Noller B (1999) Iron biominerals in medicine and the environment. Coord Chem Rev 190-192: 1199-1215 